\title{
Binary gray codes with long bit runs
}

\author{
Luis Goddyn* \\ Pavol Gvozdjak \\ Department of Mathematics \\ Simon Fraser University, Burnaby, BC, Canada \\ goddyn@math.sfu.ca, gvozdjak@math.sfu.ca
}

Submitted: Dec 21, 2001; Accepted: Jun 17, 2003; Published: Jun 27, 2003

MR Subject Classifications: 05C38, (05C45, 68R15)

\begin{abstract}
We show that there exists an $n$-bit cyclic binary Gray code all of whose bit runs have length at least $n-3 \log _{2} n$. That is, there exists a cyclic ordering of $\{0,1\}^{n}$ such that adjacent words differ in exactly one (coordinate) bit, and such that no bit changes its value twice in any subsequence of $n-3 \log _{2} n$ consecutive words. Such Gray codes are 'locally distance preserving' in that Hamming distance equals index separation for nearby words in the sequence.
\end{abstract}

Keywords: cyclic binary Gray code, Hamming distance preserving, Hamilton cycle, Hamilton circuit, $n$-cube, gap, spread, threshold, minimum run length.

\section{Introduction}

We use the language of graph theory, but where a circuit is a closed walk with no repeated internal vertices. A cycle is an orbit of a permutation acting on a set. The $n$-cube $Q_{n}$ is the graph whose vertices are the words of length $n$ on the alphabet $\{0,1\}$; two vertices are adjacent if they differ in exactly one coordinate. The transition of an edge $v w$ in $Q_{n}$ is the index $\delta_{v w} \in\{1,2, \ldots, n\}$ of the coordinate (or bit) in which $v$ and $w$ differ.

An $n$-bit (cyclic, binary) Gray code is a Hamilton circuit in $Q_{n}$. Frank Gray [3] described an elementary family of 'reflected' Gray codes (RGC) which has seen countless applications. Certain applications in engineering, statistics and computer science require specialized Gray codes with properties not possessed by the RGC. We refer to Savage [6] for more information on such variations. This paper is concerned with Gray codes for which any two edges which have equal transitions are well separated along the circuit.

${ }^{*}$ Research supported by the Natural Sciences and Engineering Research Council of Canada 
Formally, the minimum run length of a closed walk $W=w_{0} w_{1} \ldots w_{k-1} w_{0}$ in $Q_{n}$ is defined as

$$
\operatorname{mrl}(W)=\min \left\{\|i-j\|: \delta_{w_{i} w_{i+1}}=\delta_{w_{j} w_{j+1}}, i \neq j\right\} .
$$

Here, $\|i-j\|$ is the smaller of the least residues of $i-j$ and $j-i$ modulo $k$. (In case $W$ has no repeated transitions, we may define $\operatorname{mrl}(W)=k$.) In some papers, $\operatorname{mrl}(W)$ is called the threshold, the spread, or the gap of $W$.

Let $\operatorname{mrl}(n)$ be the maximum possible value of $\operatorname{mrl}(W)$ among all Hamilton circuits $W$ in $Q_{n}$. We write $\lg n$ for $\log _{2} n$. In Section 4 we prove our main result.

Theorem $1 \quad$ For $n \geq 2$ we have $\operatorname{mrl}(n) \geq\lfloor n-2.001 \lg n\rfloor$.

It is easy to see that $\operatorname{mrl}(n)<n$ for $n>2$, so this settles a conjecture appearing in [2]:

$$
\lim _{n \rightarrow \infty} \operatorname{mrl}(n) / n=1 .
$$

Constructions in [2] show only that $\operatorname{mrl}(n) / n \geq 2 / 3+o(1)$. An earlier construction of Evdokimov [1] proves that $\operatorname{mrl}(n) / n \geq 1 / 2$.

Gray codes with large minimum run length are used in electronic devices such as the Codacon [4] spectrograph. The 10-bit code used in the Codacon is presented as a sporadic construction in Section 5. Other applications such as the visualization of neural networks [7] take advantage of the 'local Hamming distance preservation' property of such Gray codes: a closed walk $W=\left(w_{i}\right)$ in $Q_{n}$ satisfies $\operatorname{mrl}(W) \geq m$ if and only if for $0 \leq i, j<k$,

$$
\|i-j\| \leq m \text { implies } d\left(w_{i}, w_{j}\right)=\|i-j\| .
$$

(Here $d\left(w_{i}, w_{j}\right)$ is the number of bits in which $w_{i}$ and $w_{j}$ differ.)

Two variations of our problem have been studied. The first is concerned with finding a longest circuit in $Q_{n}$ having a prespecified minimum run length $m$, where $\operatorname{mrl}(n)<$ $m<n$. This optimal length $\ell(n, m)$ is known [8] to satisfy $\ell(n, m) \geq 2^{n-\lceil m / 2\rceil}$. This is surely far from optimal; it is conjectured in [2] that $\ell(n, n-1) / 2^{n} \rightarrow 1$. The second variation imposes an additional separation requirement which provides the circuit with error-correcting capability. Given $d \leq m<n$, one typically seeks a longest circuit $W$ in $Q_{n}$ satisfying (1) and additionally

$$
\|i-j\| \geq d \text { implies } d\left(w_{i}, w_{j}\right) \geq d .
$$

Such objects are often called circuit codes. For $d=m=2$ this is the snake-in-a-box problem. We refer the interested reader to [5] and the references therein.

\section{Definitions}

A step permutation of $V\left(Q_{n}\right)$ is a permutation of $V\left(Q_{n}\right)$ such that each vertex is mapped to one of its neighbouring vertices. A list $\pi_{1}, \pi_{2}, \ldots, \pi_{k}$ of step permutations acting on (successive images of) a vertex $v$ naturally defines a walk of length $k$ in $Q_{n}$,

$$
W\left(v ; \pi_{1}, \pi_{2}, \ldots, \pi_{k}\right)=v v^{\pi_{1}} v^{\pi_{1} \pi_{2}} \ldots v^{\pi_{1} \pi_{2} \ldots \pi_{k}} .
$$


The stream induced by $\pi_{1}, \pi_{2}, \ldots, \pi_{k}$ is the set of $2^{n}$ walks

$$
S\left(\pi_{1}, \pi_{2}, \ldots, \pi_{k}\right)=\left\{W\left(v ; \pi_{1}, \pi_{2}, \ldots, \pi_{k}\right): v \in V\left(Q_{n}\right)\right\} .
$$

We say that the stream $S=S\left(\pi_{1}, \pi_{2}, \ldots, \pi_{k}\right)$ induces the permutation $\pi(S)=\pi_{1} \pi_{2} \ldots \pi_{k}$ of $V\left(Q_{n}\right)$. We can obviously concatenate any two streams $S, S^{\prime}$ to obtain a new stream $S S^{\prime}$. We may write $S^{2}$ for $S S$. Let $W(v ; S)$ denote the walk in $S$ with initial vertex $v$ and terminal vertex $v^{\pi(S)}$. Let $r(v)$ be the length of the orbit of $v$ under $\pi(S)$. Then $W\left(v ; S^{r(v)}\right)$ is a closed walk in the (concatenated) stream $S^{r(v)}$. The minimum run length $\operatorname{mrl}(S)$ of the stream $S$ is defined to be the smallest minimum run length among the closed walks in $\left\{W\left(v ; S^{r(v)}\right): v \in V\left(Q_{n}\right)\right\}$.

We sometimes identify $V\left(Q_{n}\right)$ with the vector space $\mathrm{GF}(2)^{n}$, denoting by $e_{i}$ the $i$ th standard vector. For example, $\delta_{v w}=i$ iff $v+w=e_{i}$. Two examples of step permutations are: the elementary involutions

$$
\tau_{i}: v \mapsto v+e_{i}, \quad i=1, \ldots, n
$$

and the following modification of $\tau_{i}$. If $u v w$ is a path of length two in $Q_{n}$ and $\delta_{v w}=i$, then $\tau_{i}^{(u v w)}$ is the step permutation defined by

$$
\tau_{i}^{(u v w)}=(u w) \tau_{i} .
$$

The orbit of $u$ under $\tau_{i}^{(u v w)}$ induces the 4-circuit uvwzu in $Q_{n}$, where $z=u^{\tau_{i}}$. Thus the cycle structure of $\tau_{i}^{(u v w)}$ is given by

$$
(u v w z) \prod\left\{\left(x x^{\tau_{i}}\right): x \in V\left(Q_{n}\right)-\{u, v, w, z\}\right\} .
$$

The transition sequence of a walk $W=w_{0} w_{1} \ldots w_{k}$ in $Q_{n}$ is defined to be the sequence

$$
\delta_{w_{0} w_{1}}, \delta_{w_{1} w_{2}}, \ldots, \delta_{w_{k-1} w_{k}} \text {. }
$$

Thus for closed walks, $\operatorname{mrl}(W)$ equals the smallest index separation (modulo $k$ ) between two identical entries in its transition sequence. The following might help illustrate these notions.

Example 2 Let $u v w=000100110$ be a path of length two in $Q_{3}$. Then $\delta_{u v}=1$ and $\delta_{v w}=2$. The following stream in $Q_{3}$ consists of eight walks of length four.

$$
\begin{aligned}
S & =S\left(\tau_{2}^{(u v w)}, \tau_{3}, \tau_{2}, \tau_{3}\right) \\
& =\left\{W_{1}, W_{2}, \ldots, W_{8}\right\}
\end{aligned}
$$

where

$$
\begin{array}{ll}
W_{1}=000,100,101,111,110 & W_{5}=100,110,111,101,100 \\
W_{2}=001,011,010,000,001 & W_{6}=101,111,110,100,101 \\
W_{3}=010,000,001,011,010 & W_{7}=110,010,011,001,000 \\
W_{4}=011,001,000,010,011 & W_{8}=111,101,100,110,111 .
\end{array}
$$

In this example we have $\pi(S)=(u w)=(000110)$ (the other 6 vertices are fixed points). Concatenating $S$ with itself gives rise to seven closed walks, $W_{1} W_{7}, W_{2}, W_{3}, W_{4}, W_{5}$, $W_{6}$, and $W_{8}$. All seven closed walks have minimum run length 2 . Thus $\operatorname{mrl}(S)$, being the minimum of these numbers, equals 2 . 


\section{The Construction}

We construct a Hamilton circuit in the cartesian product $Q_{a} \times Q_{b} \cong Q_{a+b}$ from a stream in $Q_{a}$ and a Hamilton circuit in $Q_{b}$. Let $(X, Y)$ be the bipartition of $V\left(Q_{a}\right)$ into words of even and odd weight.

Lemma 3 Let $S$ be a stream of length $2^{b}$ in $Q_{a}$ such that $X$ is one of the orbits of $\pi(S)$. Then $\operatorname{mrl}(a+b) \geq 2 \min \{\operatorname{mrl}(S), \operatorname{mrl}(b)\}$.

Proof: Let $S$ be as in the hypothesis, and let $w_{0} \in X$. Since $\pi(S)$ cyclically permutes the vertices in $X$, the concatenated stream $S^{2^{a-1}}$ contains the closed walk

$$
W=W\left(w_{0} ; S^{2^{a-1}}\right)=w_{0} w_{1} \ldots w_{2^{a+b-1}-1} w_{0} .
$$

This walk is a concatenation of the $2^{a-1}$ walks in $S$ which originate in $X$. That is, $X=\left\{w_{k 2^{b}}: 0 \leq k<2^{a-1}\right\}$. Step permutations map $X$ into $Y$ bijectively, so for each $j \in\left\{0, \ldots, 2^{b}-1\right\}$ we have that

$$
\left\{w_{k 2^{b}+j}: 0 \leq k<2^{a-1}\right\}= \begin{cases}X & \text { if } j \text { is even } \\ Y & \text { if } j \text { is odd }\end{cases}
$$

Let $Z=z_{0} z_{1} \ldots z_{2^{b}-1} z_{0}$ be a Hamilton circuit in $Q_{b}$ with $\operatorname{mrl}(Z)=\operatorname{mrl}(b)$. We assume that the index sets of the bits of $Q_{a}$ and $Q_{b}$ are disjoint. By merging the transition sequences of $W$ and the concatenated walk $Z^{2^{a-1}}$, we obtain the following walk in $Q_{a} \times Q_{b}$.

$$
\begin{aligned}
C= & \left(w_{0}, z_{0}\right)\left(w_{1}, z_{0}\right)\left(w_{1}, z_{1}\right)\left(w_{2}, z_{1}\right)\left(w_{2}, z_{2}\right) \ldots\left(w_{2^{b}-1}, z_{2^{b}-1}\right)\left(w_{2^{b}}, z_{2^{b}-1}\right) \\
& \left(w_{2^{b}}, z_{0}\right)\left(w_{2^{b}+1}, z_{0}\right)\left(w_{2^{b}+1}, z_{1}\right) \ldots\left(w_{2 \cdot 2^{b}-1}, z_{2^{b}-1}\right)\left(w_{2 \cdot 2^{b}}, z_{2^{b}-1}\right) \\
& \left(w_{2 \cdot 2^{b}}, z_{0}\right)\left(w_{2 \cdot 2^{b}+1}, z_{0}\right)\left(w_{2 \cdot 2^{b}+1}, z_{1}\right) \ldots\left(w_{3 \cdot 2^{b}-1}, z_{2^{b}-1}\right)\left(w_{3 \cdot 2^{b}}, z_{2^{b}-1}\right) \\
& \left(w_{3 \cdot 2^{b}}, z_{0}\right) \ldots \\
& \ldots \\
& \left(w_{\left(2^{a-1}-1\right) \cdot 2^{b}}, z_{0}\right)\left(w_{\left(2^{a-1}-1\right) \cdot 2^{b}+1}, z_{0}\right) \ldots\left(w_{2^{a-1} \cdot 2^{b}-1}, z_{2^{b}-1}\right)\left(w_{0}, z_{2^{b}-1}\right) \\
& \left(w_{0}, z_{0}\right) .
\end{aligned}
$$

It is immediate that $C$ is a closed walk of length $2^{a+b}$, and that

$$
\operatorname{mrl}(C)=2 \min \{\operatorname{mrl}(W), \operatorname{mrl}(Z)\} \geq 2 \min \{\operatorname{mrl}(S), \operatorname{mrl}(b)\} .
$$

It remains to show that $C$ is a Hamilton circuit. Let $(w, z) \in V\left(Q_{a}\right) \times V\left(Q_{b}\right)$. As $Z$ is a Hamilton circuit, there is a unique $j \in\left\{0,1, \ldots, 2^{b}-1\right\}$ such that $z=z_{j}$. By (3) there is a unique $k$ such that $w \in\left\{w_{k 2^{b}+j}, w_{k 2^{b}+j+1}\right\}$. By its construction, both pairs $\left(w_{k 2^{b}+j}, z_{j}\right),\left(w_{k 2^{b}+j+1}, z_{j}\right)$ are vertices of $C$, so $(w, z) \in V(C)$. Since $C$ has length $2^{a+b}$, it is a Hamilton circuit in $Q_{a} \times Q_{b}$.

Remark 4 The (essentially unique) 5-bit Gray code $C$ with $\operatorname{mrl}(C)=4$ is a special case of the above construction, with $a=3$ and $b=2$. Here $S$ is the stream $S\left(\pi_{1}, \pi_{2}, \pi_{3}, \pi_{4}\right)$ where the permutations are involutions defined by $\pi_{i}: w \mapsto w+e_{f_{i}(w)}(1 \leq i \leq 4)$, where

$$
f_{1}(w)=1, \quad f_{2}(w)=f_{4}(w)=2+w^{1}, \quad f_{3}(w)=3-w^{1},
$$


and $w^{1} \in\{0,1\}$ denotes the first bit of $w$. The resulting walk $W$ has transition sequence

$$
1,3,2,3,1,2,3,2,1,3,2,3,1,2,3,2 .
$$

The next two lemmas serve to construct a stream $S$ having large minimum run length while satisfying the two hypotheses of Lemma 3. The first lemma heeds the requirement on the orbits of $\pi(S)$. The second lemma shows how to adjust the length of the stream.

Lemma 5 There exists a stream $S^{\prime}$ in $Q_{a}(a \geq 2)$ having length $(a-1)\left(2^{a}-2\right)$ such that $X$ is an orbit of $\pi\left(S^{\prime}\right)$ and $\operatorname{mrl}\left(S^{\prime}\right)=a-1$.

Proof: Let $x_{0} y_{0} x_{1} y_{1} \ldots x_{2^{a-1}-1} y_{2^{a-1}-1}$ be a Hamilton path in $Q_{a}$ such that $\delta_{x_{j} y_{j}}=1$, for $0 \leq j<2^{a-1}$. For example, Gray's RGC has this property. The transition sequence of this path is $1, t_{1}, 1, t_{2}, 1, \ldots, t_{2^{a-1}-1}, 1$ where $t_{j}=\delta_{y_{j-1} x_{j}} \in\{2,3, \ldots, a\}$. For $1 \leq j<2^{a-1}$, we define the sequence of permutations

$$
P_{j}=\tau_{2}, \tau_{3}, \ldots, \tau_{t_{j}-1}, \tau_{t_{j}}^{(u v w)}, \tau_{t_{j}+1}, \ldots, \tau_{a}, \tau_{2}, \tau_{3}, \ldots, \tau_{a}
$$

where $u v w$ is the image of $x_{j-1} y_{j-1} x_{j}$ under the permutation $\tau_{2} \tau_{3} \ldots \tau_{t_{j}-1}$. We observe that any triple (uvw) appearing in (4) satisfies

$$
\delta_{u v}=\delta_{x_{j-1} y_{j-1}}=1 \text {. }
$$

For $1 \leq j<2^{a-1}$, we define the stream $S_{j}=S\left(P_{j}\right)$. Example 2 provides an example of $S_{j}$ when $a=3$ and $t_{j}=2$. Let

$$
S^{\prime}=S_{1} S_{2} \ldots S_{2^{a-1}-1}=S\left(\pi_{1}, \pi_{2}, \ldots, \pi_{(a-1)\left(2^{a}-2\right)}\right) .
$$

Each substream $S\left(\pi_{i}\right)$ has length one and consists of the set of edges $\left\{w w^{\pi_{i}}: w \in V\left(Q_{a}\right)\right\}$. By (4) and (5), the set of transitions of the edges in $S\left(\pi_{i}\right)$ is

$$
\left\{\delta_{w w^{\pi_{i}}}: w \in V\left(Q_{a}\right)\right\}=\left\{\begin{array}{cl}
\{k\} & \text { if } \pi_{i}=\tau_{k} \\
\{1, k\} & \text { if } \pi_{i}=\tau_{k}^{(u v w)} \text { for some uvw. }
\end{array}\right.
$$

For $2 \leq k \leq a$, any two occurrences of $\tau_{k}$ or $\tau_{k}^{(u v w)}$ in the sequence $\pi_{1}, \pi_{2}, \ldots, \pi_{(a-1)\left(2^{a}-2\right)}$ are separated by at least $a-1$ positions. Furthermore any two permutations having the form $\tau_{t_{j}}^{(u v w)}$ are separated by at least $a$ positions, since they occur only in the first half of each subsequence $P_{j}$. Thus by (6) we conclude that $\operatorname{mrl}\left(S^{\prime}\right)=a-1$.

Any two elementary involutions $\tau_{k}, \tau_{\ell}$ commute. Further, for any path uvw with $\delta_{v w}=k$ we have

$$
\tau_{k}^{(u v w)} \tau_{\ell}=\tau_{\ell} \tau_{k}^{\left(u^{\prime} v^{\prime} w^{\prime}\right)}
$$

where $u^{\prime} v^{\prime} w^{\prime}$ is the image of uvw under $\tau_{\ell}$. Thus with $u v w$ as in (4) we have

$$
\begin{aligned}
\pi\left(S_{j}\right) & =\tau_{2} \tau_{3} \ldots \tau_{t_{j}-1} \tau_{t_{j}}^{(u v w)} \tau_{t_{j}+1} \ldots \tau_{a} \tau_{2} \tau_{3} \ldots \tau_{a} \\
& =\tau_{t_{j}}^{\left(x_{j-1} y_{j-1} x_{j}\right)} \tau_{t_{j}} \prod_{k \neq t_{j}} \tau_{k}^{2} \\
& =\left(x_{j-1} x_{j}\right) .
\end{aligned}
$$

Thus $\pi\left(S^{\prime}\right)=\prod_{j=1}^{2^{a-1}-1}\left(x_{j-1} x_{j}\right)=\left(x_{0} x_{2^{a-1}-1} x_{2^{a-1}-2} \ldots x_{1}\right)$ and $X$ is an orbit of $\pi\left(S^{\prime}\right)$. 
Remark 6 It is interesting to minimize the length of $S^{\prime}$ in the statement of Lemma 5 while retaining the other two properties. For example, in the above construction, one can reduce the length of $S^{\prime}$ by omitting the second half of any $P_{j}$ for which $t_{j} \leq t_{j+1}$, and adjusting slightly the definition of uvw. (The permutations $\tau_{2}, \ldots, \tau_{a}$ comprising the second half of $P_{j}$ serve only as 'padding' to separate the modified permutation in $P_{j}$ from the the modified permutation in $P_{j+1}$. If $t_{j} \leq t_{j+1}$, then those two permutations will be separated by at least $a-1$ positions, even after $\tau_{2}, \ldots, \tau_{a}$ are deleted.) For example, if we use the RGC to define the sequence $\left(t_{j}\right)=(2,3,2,4,2,3,2,5, \ldots)$, then the relation $t_{j} \leq t_{j+1}$ holds for half of the values $j$. Modifying each corresponding $P_{j}$ as above results in a stream having $3 / 4$ the length of $S^{\prime}$.

Further reduction should be possible by writing $\left(x_{0} x_{1} \ldots x_{2^{a-1}-1}\right)$ as a product of fewer than $2^{a-1}-1$ involutions. For example, $(01234567)=\pi_{1} \pi_{2}$, where $\pi_{1}=(17)(26)(35)$ and $\pi_{2}=(10)(27)(36)(45)$. Of course one must then express each involution as a product of just a few step permutations, while maintaining the minimum run length requirement.

Lemma 7 For any even integer $\ell \geq(a-1)\left(2^{a}+2 a-6\right)$, there exists a stream $S$ in $Q_{a}$ $(a \geq 2)$ having length $\ell$ such that $X$ is an orbit of $\pi(S)$ and $\operatorname{mrl}(S)=a-1$.

Proof: Let $\ell=(a-1)\left(2^{a}-2\right)+2 t$ where $t \geq(a-1)(a-2)$. It is a standard result that there exist nonnegative integers $\alpha$ and $\beta$ such that

$$
t=\alpha a+\beta(a-1) .
$$

Let $S^{\prime}, P_{j}$ and $S_{j}$ be as in Lemma 5 , and consider the stream $S=S^{\prime} T^{2 \alpha} R^{2 \beta}$ where $T=S\left(\tau_{1}, \tau_{2}, \ldots, \tau_{a}\right)$ and $R=S\left(\tau_{2}, \tau_{3}, \ldots, \tau_{a}\right)$. It is immediate that $S$ has length $\ell$ and $\pi(S)=\pi\left(S^{\prime}\right)$. It remains to show that appending $T^{2 \alpha} R^{2 \beta}$ does not decrease the minimum run length of $S^{\prime}$. Since $\operatorname{mrl}\left(T^{2 \alpha} R^{2 \beta}\right)=a-1$, we need only check the 'boundary substreams'. It is possible that $\alpha$ or $\beta$ equals zero, so we should verify that each of the four streams $T S_{1}, R S_{1}, S_{2^{a-1}-1} T, S_{2^{a-1}-1} R$, has minimum run length at least $a-1$. By (4) and (6) this amounts to verifying that the permutations $\tau_{1}$ and $\tau_{t_{j}}^{(u v w)}$ are separated by at least $a-1$ positions in the permutation sequences $\tau_{1}, \tau_{2}, \ldots, \tau_{a}, P_{j}$ and $P_{j}, \tau_{1}, \tau_{2}, \ldots, \tau_{a}$. Thus we prove $\operatorname{mrl}(S)=a-1$.

Putting this together with Lemma 3 yields our basic recurrence.

Corollary 8 If $(a-1)\left(2^{a}+2 a-6\right) \leq 2^{b}$, then

$$
\operatorname{mrl}(a+b) \geq 2 \min \{a-1, \operatorname{mrl}(b)\} .
$$

Starting with known lower bounds on $\operatorname{mrl}(n)$ for small $n$ as given in Section 5 , it is easy to write a computer program that applies (7) in an optimal manner for $n \leq 2000$. The result (Figure 1) suggests that $\operatorname{mrl}(n) \geq n-2 \lg n$ for all $n$. In the next section we prove a bound which is almost this good. 


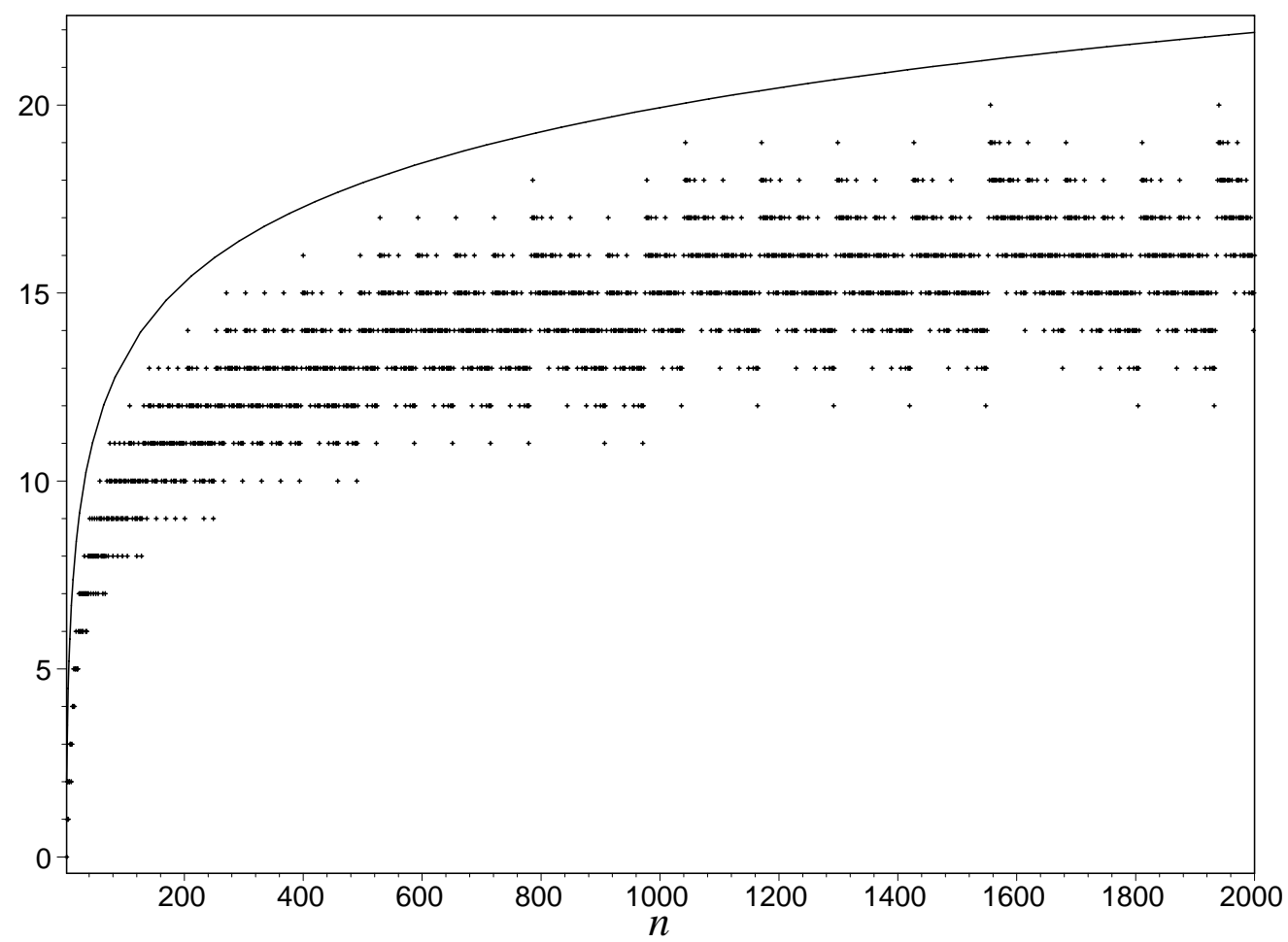

Figure 1: Comparing $2 \lg n$ with the upper bound on $n-\operatorname{mrl}(n)$ arising from (7).

\section{Proof of Theorem 1}

We show that, for any $c>2$, if the inequality

$$
\operatorname{mrl}(n) \geq\lfloor n-c \lg n\rfloor
$$

holds for all $n \geq 2$ satisfying

$$
\left(2-\frac{2 c \lg n}{n}\right)^{c}<4 .
$$

then (8) holds for all $n \geq 2$. Let $N(c)$ denote the greatest integer $n$ for which (9) holds. For example, $N(3)=95$. (The function $x \mapsto N(2+1 / x)$, plotted in Figure 2, is slightly superlinear.) Let $n>N(c)$. There exists an integer $b \geq n / 2$ such that $a+b \leq n \leq a+b+1$, where

$$
a=b-\lfloor c \lg b\rfloor+1 \text {. }
$$

We first observe

$$
\frac{a+b}{b} \geq 2-\frac{c \lg b}{b} \geq 2-\frac{c \lg (n / 2)}{n / 2} \geq 2-\frac{2 c \lg n}{n} \geq 4^{1 / c},
$$

and verify that

$$
(a-1)\left(2^{a}+2 a-6\right) \leq a 2^{a} \leq 2 a 2^{b} / b^{c} \leq 2^{b} .
$$




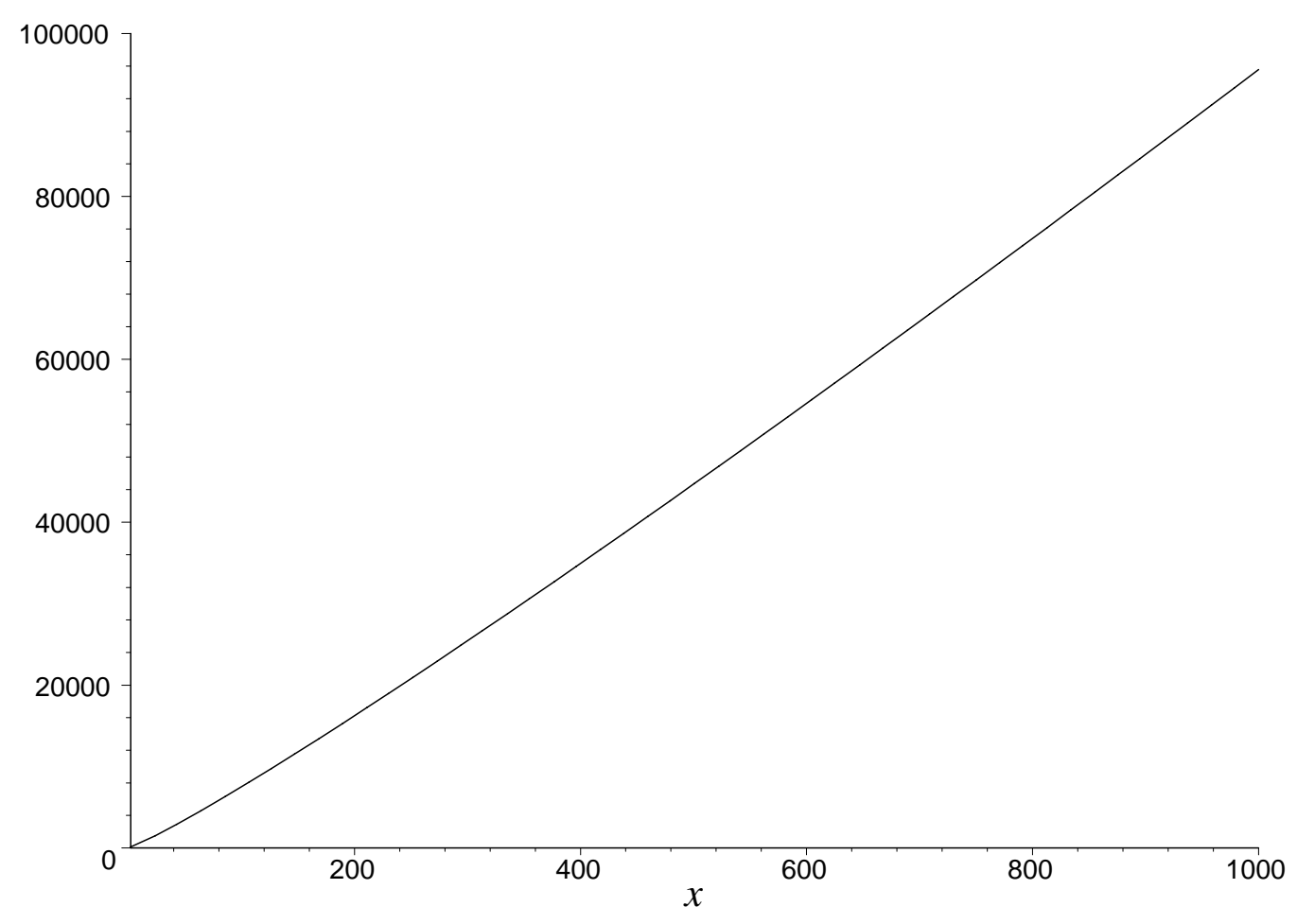

Figure 2: $N(2+1 / x)$ versus $x$.

Using the elementary fact $\operatorname{mrl}(n) \geq \operatorname{mrl}(n-1)$, we apply Corollary 8 inductively as follows.

$$
\begin{aligned}
\operatorname{mrl}(n) & \geq \operatorname{mrl}(a+b) \\
& \geq 2 \min (a-1, \operatorname{mrl}(b)) \\
& \geq 2 \min (b-\lfloor c \lg b\rfloor, b-\lfloor c \lg b\rfloor) \\
& =a+b-\lfloor c \lg b\rfloor-1 \\
& =a+b+1-\left\lfloor c \lg (a+b)+c \lg \left(4^{1 / c} b /(a+b)\right)\right\rfloor \\
& \geq n-\lfloor c \lg n\rfloor .
\end{aligned}
$$

Using (7) to electronically verify (8) for $n \leq 10^{5}$, we find that (8) holds for all $n$ when $c=2.001$.

\section{$5 \quad$ Small $n$ and a Sporadic Construction}

The effective application of the construction in Section 3 depends heavily on good lower bounds on $\operatorname{mrl}(n)$ for small values of $n$. The following table summarizes the best lower bounds $\ell(n)$ that can be achieved by the constructions in [2], and the two sporadic constructions described below. By use of exhaustive computer searches, one can show that 
the starred values are exact.

\begin{tabular}{r|llllllllrrrrrrrrrrrr}
$n$ & 2 & 3 & 4 & 5 & 6 & 7 & 8 & 9 & 10 & 11 & 12 & 13 & 14 & 15 & 16 & 17 & 18 & 19 & 20 & 21 \\
\hline$\ell(n)$ & $2^{*}$ & $2^{*}$ & $2^{*}$ & $4^{*}$ & $4^{*}$ & $5^{*}$ & 5 & 6 & 8 & 8 & 8 & 9 & 9 & 11 & 11 & 12 & 12 & 14 & 15 & 16
\end{tabular}

We now describe a hitherto unpublished construction which yields a 10-bit Gray code with minimum run length 8 . This is the Gray code used in the Codacon device [4]. The construction in Section 3 and those given in [1,2] can only produce 10-bit codes with minimum run length at most 7.

Let $C_{4}$ denote the circuit of length four. We view the 10-cube as a cartesian power

$$
Q_{10} \cong G=C_{4} \times C_{4} \times C_{4} \times C_{4} \times C_{4}
$$

We identify each vertex of $G$ with a group element $\left(a_{0}, a_{1}, a_{2}, a_{3}, a_{4}\right) \in Z_{4}^{5}$, where $Z_{4}$ is the integers modulo 4. Two vertices $u, v$ are adjacent in $G$ if $u-v= \pm e_{i}$, where $e_{i}$ is the $i$ th unit group element, $0 \leq i \leq 4$. A $G$-transition sequence is any word $T=t_{1} t_{2} \ldots t_{k}$ from the alphabet $\{0,1,2,3,4\}$. As usual, if $v \in V(G)$, then $T$ induces a walk $W(v ; T)=$ $v_{0} v_{1} \ldots v_{k}$ in $G$ with $v_{0}=v$ and $v_{i}=v_{i-1}+e_{t_{i}}$. Let $T^{i \mapsto 4}$ be the set of words that can be obtained from $T$ by replacing one copy of the symbol $i$ by the symbol 4 . For example, $(0123401)^{1 \mapsto 4}=\{0423401,0123404\}$.

Let $T_{0}=0123012301230123$. We iteratively (and nondeterministically) define $G$ transition sequences $T_{1}, T_{2}$ and $T_{3}$ by

$$
T_{i+1}=T_{i}^{\prime} T_{i}^{\prime} T_{i}^{\prime} T_{i}^{\prime}, \text { where } T_{i}^{\prime} \in T_{i}^{i \mapsto 4} .
$$

Each $W\left(v ; T_{i}\right)$ is a closed walk of length $4^{i+2}$. It can be checked by computer, or proved with some effort, that any walk $W\left(v ; T_{i}\right)(0 \leq i \leq 3)$ is a circuit in $G$. Thus $W\left(v ; T_{3}\right)$ represents a 10-bit Gray code $C$.

The minimum run length of $C$ is at least twice the minimum distance between two occurrences of the same symbol in $T_{3}$. For $j \in\{0,1,2,3\}$, two occurrences of symbol $j$ in $T_{3}$ are separated by at least four positions. By selecting the sequences $T_{i}^{\prime}$ sensibly, the same statement holds for $j=4$. Thus $\operatorname{mrl}(10) \geq \operatorname{mrl}(C) \geq 8$.

This construction does not appear to easily generalize to higher dimensional cubes. A similar construction using the subgraph $C_{4}^{7} \times C_{8}^{2} \subseteq Q_{20}$ and $T_{0}=(01234567)^{8}$. can be made to produce a noncyclic 20-bit Gray code with minimum run length 16 . If $T$ is a word in the alphabet $\{0,1, \ldots, 19\}$ representing its transition sequence, then $T(20) T(20)$ represents a 21-bit cyclic Gray code, whence $\operatorname{mrl}(21) \geq 16$. We omit details here. Other, more complicated schema have produced minor improvements that are not worth mentioning here. We leave as unsolved the problem of generalizing this 10-bit construction in a satisfactory manner.

\section{Acknowledgment}

We thank D. Knuth for providing helpful comments regarding the proof of Theorem 1. 


\section{References}

[1] A. A. Evdokimov, Numeration of subsets of a finite set. (Russian) Metody Diskret. Analiz. 34, (1980), 8-26, 100.

[2] L. Goddyn, G. M. Lawrence, E. Nemeth, Gray Codes with Optimized Run Lengths, Utilitas Mathematica 34 (1988), 179-192.

[3] F. Gray, Pulse Code Communication, U. S. Patent No. 2632058, (March 15, 1953).

[4] G. M. Lawrence, W. E. McClintlock, Compact Ultraviolet Imaging Microchannel Plate Detectors using CODed Anode CONverter (CODACON) Readout Systems, SPIE Proceedings Vol. 2831, The International Society of Optical Engineering, Robert E. Huffman, Ed., 1996, ISBN 0-8194-2219-3, pp. 104-111.

[5] Kenneth G. Paterson, Jonathan Tuliani, Some new circuit codes, IEEE Trans. Inform. Theory 44 (1998), 1306-1309.

[6] Carla Savage, A survey of combinatorial Gray codes, SIAM Rev. 39 (1997), 605-629.

[7] Colin E. Thorne, "The structure and construction of super Gray codes," Masters of Applied Science Candidate Thesis, Queensland University of Technology, Griffith Campus, 1993.

[8] A. J. van Zanten, Agung Lukito, Construction of certain cyclic distance-preserving codes having linear-algebraic characteristics. Des. Codes Cryptogr. 16 (1999), 185199. 